\title{
Three Metachronous Cases of HER2-Positive Breast Cancer Accompanied with Thyroid Cancer
}

\author{
Qiqi Gao Yulong Zheng Bo Wang Zihan Wu Guoping Ren
}

The first Affiliated Hospital, School of Medicine, Zhejiang University, Hangzhou, China

\section{Established Facts}

- PTC is common among women with BC.

- Collision metastases in lymph nodes do occur but are not common.

\section{Novel Insights}

- HER2-positive breast cancer may be at a higher risk for thyroid cancer than other cancers.

- Finding 2 distinct types of cancer metastasized to 1 lymph node in a patient may, therefore, warrant a high index of suspicion and the use of immunohistochemical stains to differentiate the types of collision tumors.

\section{Keywords}

Collision metastasis - Breast cancer · Immunohistochemistry · Papillary thyroid carcinoma · HER2

\section{Summary}

Background: Breast carcinoma $(\mathrm{BC})$ and papillary thyroid carcinoma (PTC) occur predominantly in women. Many studies have shown that PTC is common among women with BC. Collision metastases in lymph nodes do occur but are not common. Case Report: Here we report 3 metachronous cases of BC with PTC. PTC developed after $B C$ in each case. Histology and immunohistochemistry revealed that HER2 status was positive in each $B C$, and in 1, concurrent metastases of BC and PTC were shown in a lymph node of the left cervical region. Conclusion: From our results, we speculate that women with HER2positive breast cancer may be at a higher risk for thyroid cancer than other cancers. Finding 2 distinct types of cancer metastasized to 1 lymph node in a patient may, therefore, warrant a high index of suspicion and the use of immunohistochemical stains to differentiate the types of collision tumors.

\section{Introduction}

Papillary thyroid carcinoma (PTC) and breast carcinoma (BC) may occur concomitantly or successively in middle-aged females. Both carcinomas tend to metastasize along the lymphatic vessels [1]. Collision metastases in lymph nodes do occur but are rare. Here we present 3 metachronous cases of HER2-positive breast cancer with thyroid cancer, 1 with collision metastasis to a single cervical lymph node.

\section{KARGER \\ Fax +497614520714 \\ Information@Karger.com}

www.karger.com

\section{(c) 2014 S. Karger GmbH, Freiburg}

$1661-3791 / 14 / 0095-0360 \$ 39.50 / 0$

Accessible online at:

www.karger.com/brc 


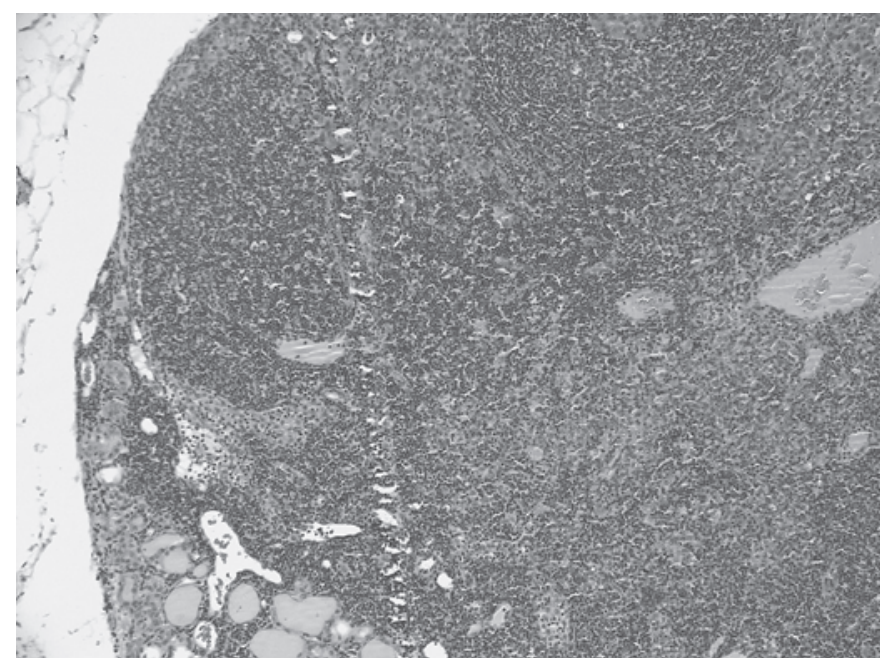

Fig. 1. Metastatic breast invasive ductal carcinoma and papillary thyroid carcinoma in the right cervical lymph node (hematoxylin and eosin, $100 \times)$.

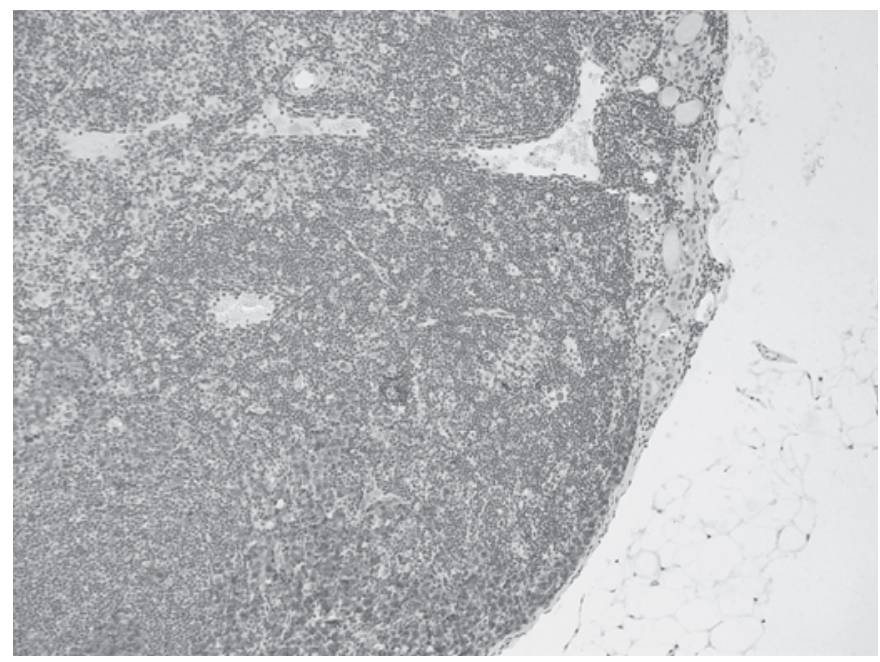

Fig. 3. Immunostaining for HER2 in the lymph node. Metastatic breast invasive ductal carcinoma (lower left corner) was positive. The metastatic papillary thyroid carcinoma (upper right corner) was negative (100×).

\section{Case Report 1}

A 59-year-old postmenopausal female patient was admitted to our hospital because of a left breast lump with pain. The biopsy showed infiltrating ductal carcinoma. After 6 cycles of chemotherapy with docetaxel/ epirubicin/cyclophosphamide, the lesion in the breast decreased a little in size. She underwent a modified radical mastectomy (MRM) of the left breast and the axillary lymph node was removed. Histopathological examination confirmed the lesion as an invasive ductal carcinoma (grade III), with regional lymph node metastasis (staging T2N2M0). Immunostaining was positive for estrogen receptors (ERs), progesterone receptors (PRs), E-cadherin (E-ca) and HER2, but negative staining for p53 and cytokeratin 5 (CK5). After 1 cycle of chemotherapy with docetaxel/ epirubicin/cyclophosphamide, the patient presented with nodules in the bilateral thyroid on palpation at physical examination. Serum triiodothyronine (T3), thyroxine (T4) and thyroid-stimulating hormone (TSH) values were all within the normal range. Ultrasound examination showed an

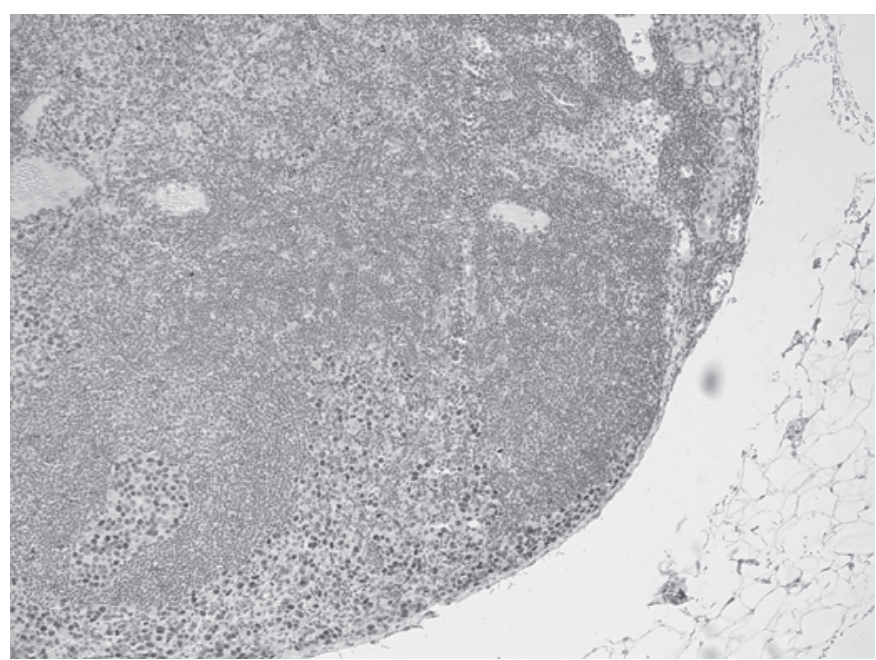

Fig. 2. Immunostaining for estrogen receptor of the metastatic breast invasive ductal carcinoma in the lymph node (lower left corner) was positive. The metastatic papillary thyroid carcinoma (upper right corner) was negative (100x).

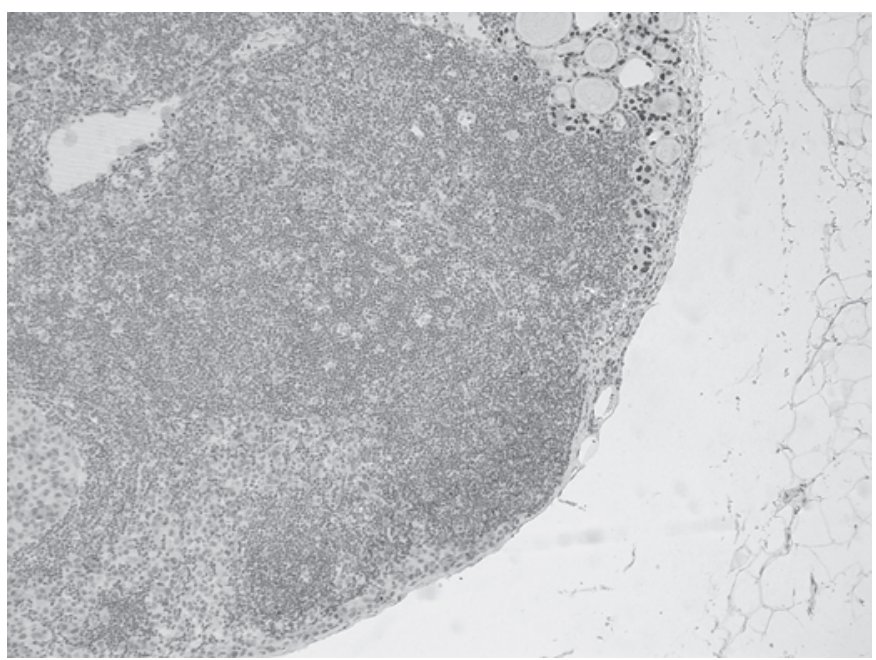

Fig. 4. Immunostaining for thyroid transcription factor- 1 in the lymph node. The metastatic breast invasive ductal carcinoma (lower left corner) was negative. The metastatic papillary thyroid carcinoma (upper right corner) was positive $(100 \times)$.

ill-defined mass in the left lobe and several colloid nodules in right lobe. She underwent a total thyroidectomy with bilateral neck lymph nodes dissection. 5 lymph nodes measuring $0.5-6 \mathrm{~mm}$ in diameter were found; among these, 3 were found to contain metastases from BC. Notably, 1 lymph node revealed a focus of both metastatic BC and PTC with each component maintaining a distinct immunophenotypic profile (fig. 1). Immunostaining for ER (fig. 2), PR, HER2 (fig. 3), E-ca, CK5, thyroglobin (TG) and thyroid transcription factor-1 (TTF-1) (fig. 4) was performed on the primary and the lymph node-metastatic breast and thyroid samples. The morphology of primary and metastatic $\mathrm{BC}$ showed poorly differentiated cells with many necrosis (fig. 5).

The breast primary and the morphologically similar metastatic deposits were positive for ER, PR, HER2 and E-ca, and negative for CK5, TG and TTF-1. The thyroid primary and metastatic PTC was made up of follicles lined by columnar cells with overlapping nuclei, which showed a typical ground-glass appearance and nuclear grooves. The metastatic deposits, which were similar morphologically to the PTC, were also immu- 
nophenotypically similar to the corresponding primary (positive for TG and TTF-1, negative for ER, PR, HER2, E-ca and CK5).

\section{Case Report 2}

A 54-year-old premenopausal female patient was admitted to our hospital because of a right breast mass with occasional pain. On examination, a fixed, firm-to-hard lump of $4 \times 3 \mathrm{~cm}$ in the upper outer quadrant of the right breast was found; no enlarged lymph nodes were palpable. Ultrasound showed an inhomogeneous low-level echo of $8 \times 1.4 \mathrm{~cm}$ in the upper outer quadrant of right breast. Intraoperatively, a sharply defined and hard lump of $7.0 \times 5.0 \mathrm{~cm}$ was found. Analysis of the sections of the intraoperatively frozen mass confirmed the diagnosis of high-grade intraductal carcinoma. The patient underwent an MRM of the right breast, and the axillary lymph node was removed. Histopathological examination confirmed the lesion as an invasive ductal carcinoma (grade II) without lymph node metastasis (staging T4NOM0). Immunostaining was positive for ER, E-ca and HER2, but negative staining for PR and p53. She underwent 6 cycles of chemotherapy with docetaxel/cyclophosphamide. 10 months after operation, the patient presented with a nodule of $0.5 \times 0.6 \mathrm{~cm}$ in the right thyroid revealed by ultrasound. Serum T3, T4 and TSH values were all within the normal range. The intraoperatively frozen sections of this nodule confirmed the diagnosis of PTC. She underwent a total thyroidectomy with bilateral neck lymph node dissection. Histopathological examination confirmed that there were no lymph node metastases.

\section{Case Report 3}

A 49-year-old premenopausal female patient was admitted to our hospital because of a right breast mass. On examination, a fixed, firm-to-hard lump of $4 \times 3 \mathrm{~cm}$ was found in the upper outer quadrant of right breast; no enlarged lymph nodes were palpable. Ultrasound showed a low-level echo of $4 \times 3 \mathrm{~cm}$ in the upper outer quadrant of right breast. Intraoperatively, an ill-defined and hard lump of $1.0 \times 1.0 \mathrm{~cm}$ was found. Examination of the sections of the intraoperatively frozen mass confirmed the diagnosis of invasive ductal carcinoma. The patient underwent an MRM of the right breast, and the axillary lymph node was removed. Histopathological examination confirmed the lesion as an invasive ductal carcinoma (grade II) without lymph node metastasis (staging T1cN0M0). Immunostaining was positive for ER, E-ca, PR and HER2, and p53. She underwent 6 cycles of chemotherapy with cyclophosphamide/epirubicin/5fluoruracil. 2 years 9 months after operation, the patient presented with a nodule of $0.7 \times 0.6 \mathrm{~cm}$ in the left thyroid revealed by ultrasound. Serum T3, T4 and TSH values were all within the normal range. Examination of the intraoperatively frozen sections of this nodule confirmed the diagnosis of PTC. She underwent a total thyroidectomy with bilateral neck lymph node dissection. Histopathological examination confirmed that there were no lymph node metastases.

\section{Discussion}

Both thyroid and breast cancers are common in females and the coincidence of thyroid cancer and breast cancer is not rare [2]. Several studies have demonstrated an increased risk of thyroid cancer in patients with breast cancer [3, 4]. Thyroid and breast are both under the influence of similar hormones. TSH receptors are present abundantly in normal breast tissue

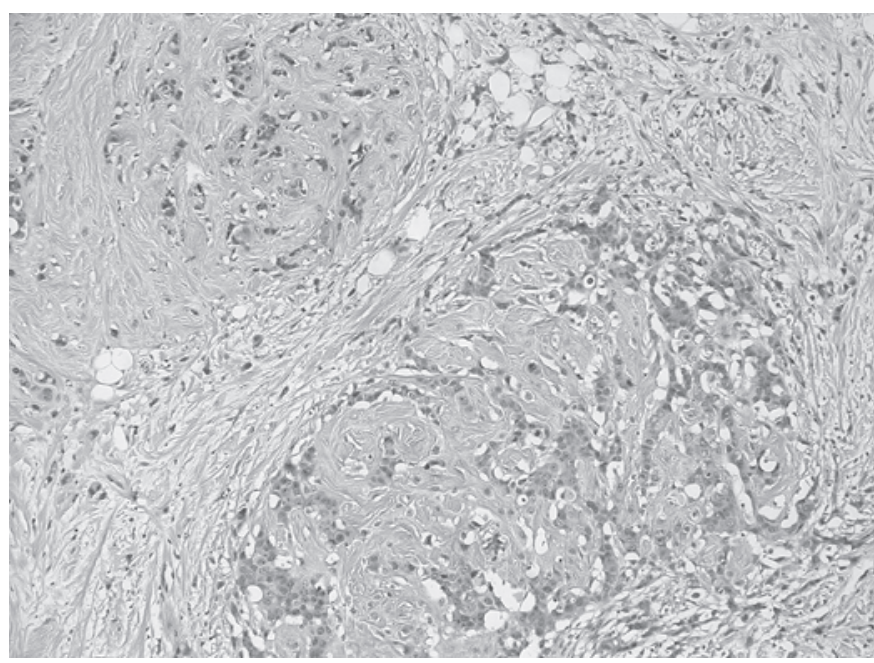

Fig. 5. The morphology of primary breast carcinoma shows poorly differentiated cells with many necrosis $(100 \times)$.

and breast cancer [5]. On the other hand, ER and PR are present in thyroid tumor cells, which suggests that female sex hormones may affect the biology of thyroid neoplasms $[6,7]$. Geographic variations of the incidence of breast cancer have been suggested to be a result of differences in dietary iodine intake [8]. Previous studies have found that activation of Akt or mutations in CHEK2 appeared to play an important role in the tumorigenesis of both thyroid and breast cancers $[9,10]$. Bardhan et al. [11] reported 2 male patients with HER2-positive breast cancer who also developed thyroid cancer, and speculated that women with HER2-positive breast cancer may have a higher risk for thyroid cancer. Zeng et al. [1] also reported a metachronous HER2-positive breast cancer with thyroid cancer. In other studies reporting a coincidence between breast cancer and thyroid disorders, no reference was made as to whether the BC was HER2 positive $[12,13]$. The present 3 cases all showed positive staining for HER2. Of a series of 276 women with HER2-positive BC diagnosed between 2009 and 2012 at our hospital, 271 patients had no other tumors; 3 suffered PTC, 2 intestinal cancer within a short period. This finding provokes speculation as to whether women with HER2-positive breast cancer are at higher risk for thyroid cancer than for other cancers (e.g. endometrial cancer, ovarian cancer, etc.). However, this should be verified by more cases. Collision metastases of carcinoma from 2 separate primary lesions to the same node are rare [14]. To our knowledge, our Case 1 is the second case of lymph node collision metastasis between breast cancer and thyroid cancer [1]. D'Andrea et al. [15] demonstrated a high grade of correlation between primary $\mathrm{BC}$ and matched lymph node metastasis in terms of immunohistochemical expression of ER, PR and HER2. In our case, the tumor of corresponding metastatic node had a similar profile to the primary BC. Both showed positive staining for HER2, ER and PR. Distinguishing the profile of metastatic tumors has an important role in selecting 
the optimal management and judging prognosis. Collision tumors are clinically relevant in that the individual tumors may require different treatments. Most collision tumors are thought to carry a poor prognosis. This poor prognosis is dependent on the biological behavior of each original tumor and on the progress of the disease, irrespective of the collision in different nodes [16].

\section{Conclusion}

An association between BC and PTC has been suggested by some investigators, and our study illustrates that PTC may occur in women with HER2-positive BC. The coincidence of 2 cancers is not rare. When evaluating lymph nodes in a pa- tient diagnosed with 2 distinct types of cancer, a high index of suspicion is warranted, and immunohistochemical staining should be used to differentiate types of collision tumors.

\section{Acknowledgments}

The authors thank the Professors at the Department of Pathology (The First Affiliated Hospital of Zhejiang University, Hangzhou, China) for their consultation on this case.

\section{Disclosure Statement}

The authors declare no conflict of interest.

\section{References}

1 Zeng $\mathrm{H}$, Liu C, Zeng YJ, et al.: Collision metastasis of breast and thyroid carcinoma to a single cervical lymph node: Report of a case. Surg Today 2012;42 891-894.

2 Agarwal DP, Soni TP, Sharma OP, et al.: Synchronous malignancies of breast and thyroid gland: A case report and review of literature. J Cancer Res Ther 2007:3:172-173.

-3 Ron E, Curtis R, Hoffman D, et al.: Multiple primary breast and thyroid cancer. Br J Cancer 1984 49:87-92.

4 Volk N, Pompe-Kirn V: Second primary cancers in breast cancer patients in Slovenia. Cancer Causes Control 1997;8:764-770.

$\checkmark 5$ Turken O, NarIn Y, DemIrbas S, et al.: Breast cancer in association with thyroid disorders. Breast Cancer Res 2003;5:R110-113.

6 Van Hoeven K, Menendez-Botet C, Strong E, et al.: Estrogen and progesterone receptor content in human thyroid disease. Am J Clin Pathol 1993;99: 175-181.
Tavangar S, Monajemzadeh M, Larijani B, et al.: Immunohistochemical study of oestrogen receptors in 351 human thyroid glands. Singapore Med J 2007;48:744-747.

8 Smyth PP: The thyroid, iodine and breast cancer. Breast Cancer Res 2003;5:235-238.

9 Kada F, Saji M, Ringel MD: Akt: A potential target for thyroid cancer therapy. Curr Drug Targets Immune Endocr Metabol Disord 2004;4:181-185.

10 Cybulski C, Górski B, Huzarski T, et al.: CHEK2 is a multiorgan cancer susceptibility gene. Am J Hum Genet 2004;75:1131-1135.

11 Bardhan P, Bui MM, Minton S, et al.: HER2-positive male breast cancer with thyroid cancer: An institutional report and review of literature. Ann Clin Lab Sci 2012;42:135-139.

12 Huang J, Walker R, Groome PG, et al.: Risk of thyroid carcinoma in a female population after radiotherapy for breast carcinoma. Cancer 2001;92: 1411-1418.
3 Vassilopoulou-Sellin R, Palmer L, Taylor S, et al.: Incidence of breast carcinoma in women with thyroid carcinoma. Cancer 1999;85:696-705.

14 Mattioli F, Masoni F, Ponti G, et al.: „Collision“ metastasis from unknown primary squamous cell carcinoma and papillary microcarcinoma of thyroid presenting as lateral cervical cystic mass. Auris Nasus Larynx 2009;36:372-375.

15 D'Andrea MR, Limiti MR, Bari M, et al.: Correlation between genetic and biological aspects in primary non-metastatic breast cancers and corresponding synchronous axillary lymph node metastasis. Breast Cancer Res Treat 2007;101:279-284.

16 Bhavsar T, Liu J, Huang Y: Collision metastasis of urothelial and prostate carcinomas to the same lymph node: A case report and review of the literature. J Med Case Rep 2012;6:124. 